\title{
Evaluation of the added mass for a spheroid-type unmanned underwater vehicle by vertical planar motion mechanism test
}

\author{
Seong-Keon Lee ${ }^{1}$, Tae-Hwan Joung ${ }^{1}$, Se-Jong Cheon ${ }^{2}$, Taek-Soo Jang ${ }^{1}$ and Jeong-Hee Lee ${ }^{1}$ \\ ${ }^{I}$ Department of Naval Architecture and Ocean Engineering, Pusan National University, Busan, Korea \\ ${ }^{2}$ Department of Offshore Engineering, Samsung Heavy Industry, Geo-je, Korea
}

\begin{abstract}
This paper shows added mass and inertia can be acquired from the pure heaving motion and pure pitching motion respectively. A Vertical Planar Motion Mechanism (VPMM) test for the spheroid-type Unmanned Underwater Vehicle (UUV) was compared with a theoretical calculation and Computational Fluid Dynamics (CFD) analysis in this paper. The VPMM test has been carried out at a towing tank with specially manufactured equipment. The linear equations of motion on the vertical plane were considered for theoretical calculation, and CFD results were obtained by commercial CFD package. The VPMM test results show good agreement with theoretical calculations and the CFD results, so that the applicability of the $V P M M$ equipment for an underwater vehicle can be verified with a sufficient accuracy.
\end{abstract}

KEY WORDS: Unmanned Underwater Vehicle (UUV); Vertical Planar Motion Mechanism test (VPMM); Added mass and inertia; Lamb's k-factors; CFD analysis.

\section{INTRODUCTION}

Recently, UUVs are increasingly used, among in scientific, industrial and military applications. Before designing an UUV, the numerical simulations with a mathematical model are needed to test its performance. The mathematical model contains a number of hydrodynamic derivatives including added mass and damping coefficients. For the precise design of UUVs, therefore, the accurate values of the derivatives are required.

The hydrodynamic derivatives can be obtained by several experimental techniques which involve a planar motion mechanism (PMM) test, a rotating arm test and a circular motion test (CMT). Among the above, the most popular is a PMM test, which has the advantage that can acquire both added masses and damping forces simultaneously.

In this study, to verify the VPMM equipment for an underwater vehicle, the PMM test for a spheroid-type UUV is performed in a towing tank. The experimental results are compared with the theoretical values (Lamb and Sir Horace, 1945) and the results from CFD analysis.

\section{VPMM TEST}

\section{VPMM Equipment}

For the purpose of a VPMM test for underwater vehicles,

Corresponding author: Tae-Hwan Joung

e-mail: s_joung@cnu.ac.kr the VPMM equipment is developed as following as Fig. 1 and specifications are shown in Table 1.

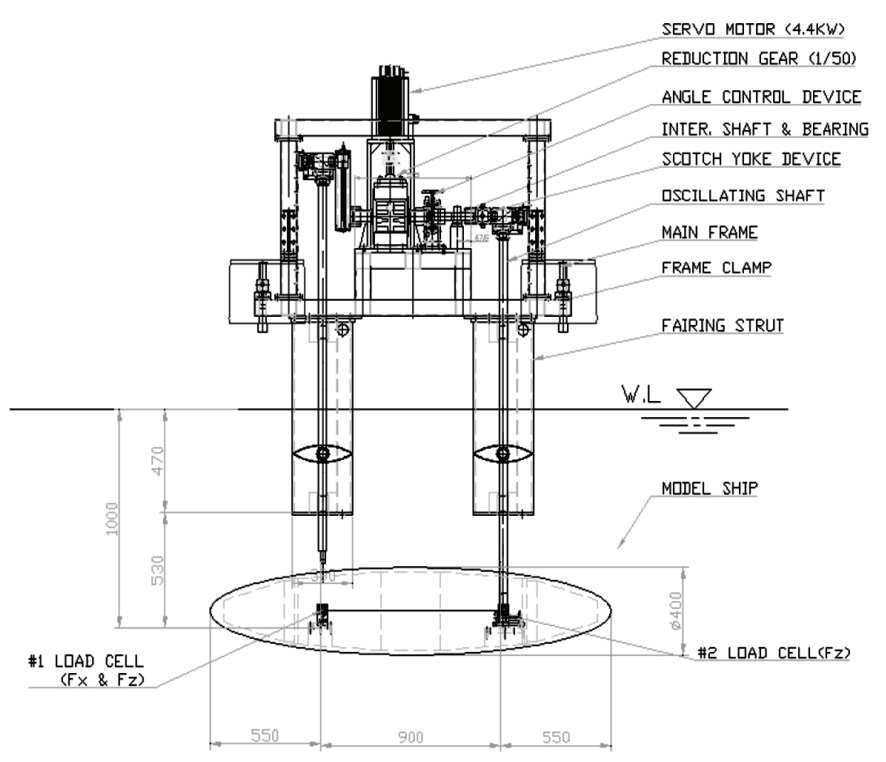

Fig. 1 VPMM equipment.

\section{Test Procedure}

The Spheroid-type model is adopted to compare the experimental results with theoretical values and CFD results. 
Table 1 Specifications of VPMM equipment.

\begin{tabular}{|c|c|}
\hline Item & Specification \\
\hline Test model & $\mathrm{L}=$ approx. $2 \mathrm{~m}$ \\
\hline Test speed & $\mathrm{U}=$ approx. $1.0 \mathrm{~m} / \mathrm{s}$ \\
\hline Pure heave motion & Approx. $\pm 0.25 \mathrm{~m}$ \\
\hline Pure pitch motion & Approx. $\pm 15 \mathrm{Deg}$. \\
\hline Strut type & 2-streamline shape strut \\
\hline Control motor & Servo motor 1set \\
\hline Reduction motor & Worm reducer \\
\hline Control system & Speed control \\
\hline 2-Comp. load cell & $\mathrm{Fz}=1000 \mathrm{~N}, \mathrm{Fx}=500 \mathrm{~N}$ \\
\hline 1-Comp. load cell & $\mathrm{Fz}=1000 \mathrm{~N}$ \\
\hline
\end{tabular}

The particulars of the model are given in Fig. 2. The coordinate system adopted in Fig. 3. The forces $\mathrm{X}$ and $\mathrm{Z}$ were measured by using one- and two- component load cells set up on the fore and the aft of the model, respectively. The moment $\mathrm{M}$ can be calculated as

$M=z_{2} l_{2}-z_{1} l_{1}$

Where, $l_{1}$ and $l_{2}$ are the distance from the center of gravity (C.G.) and subscripts 1, 2 indicate the fore and the aft, respectively.

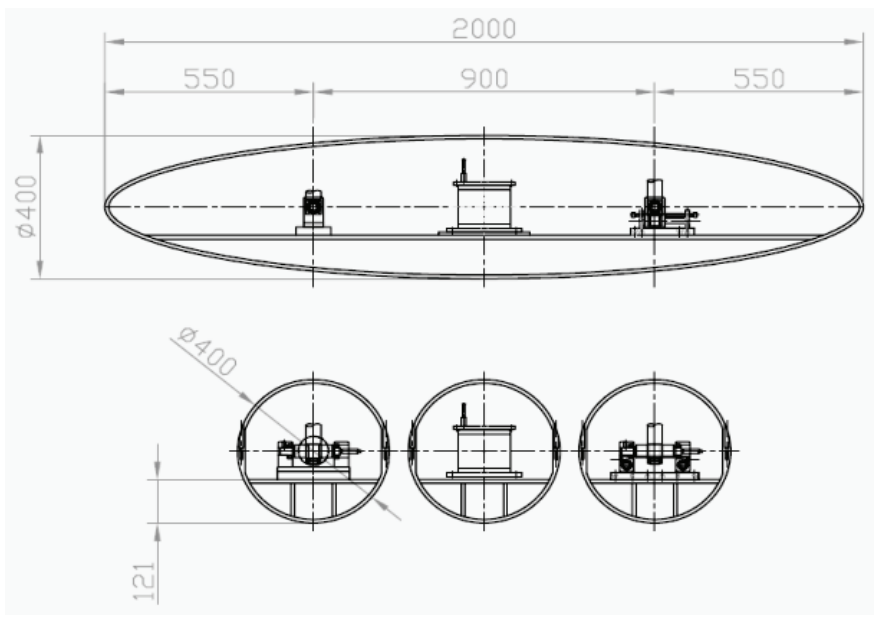

Fig. 2 Spheroid-Type UUV test model.

$\mathrm{O}$

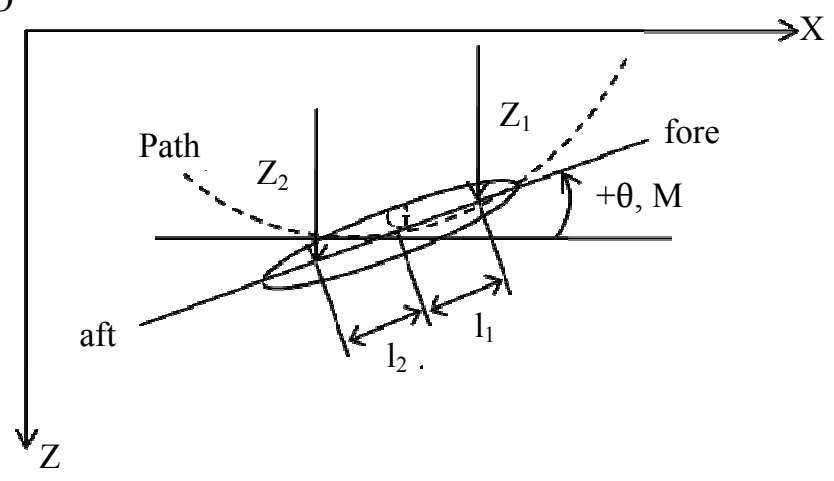

Fig. 3 Coordinate system.
In pure heaving case, the forced oscillating periods are $5,8,10 \mathrm{~s}$ and heaving amplitude is about $150 \mathrm{~mm}$. In pure pitching case, the forced oscillating period is 10 , $12 s$ and pitching amplitude is about $5.4^{\circ}$. Both cases were tested at $0.7 m$-deep which was the maximum depth with the equipment. The velocity of towing carriage is about $1 \mathrm{~m} / \mathrm{s}$. All of data were sampled at a rate of $100 \mathrm{~Hz}$ for about $50 \mathrm{~s}$.

\section{Mathematical Model}

6-DOF (Degree Of Freedom) mathematical equations of motion for underwater vehicles (Abkowitz, 1969) only about vertical motion (pitching and heaving motion) can be written as following.

$Z=m\left[\dot{w}+p v-q u-z_{G}\left(p^{2}+q^{2}\right)+x_{G}(r p-\dot{q})\right]$

$M=I_{y y} \dot{q}+\left(I_{x x}-I_{z z}\right) r p+$

$m\left[z_{G}(\dot{u}+q w-r v)-x_{G}(\dot{w}+p v-q u)+x_{G} z_{G}\left(p^{2}-r^{2}\right)\right]$

Considering the coupled motion between pitching and heaving motion and taking the linear terms in each equation (Shon et al., 2006), the linear equations for vertical motion can be obtained.

In the case of heaving motion, the forces measured have opposite sign to the motion. That is, when the model is downward, positive $z$-direction, the negative forces are measured. Therefore in the right-hand side of Eq. (2), the external force $Z_{E}$ has negative sign.

- Linear equation of heaving motion:

$-Z_{E}=\left(m+A_{33}\right) \dot{w}-Z_{w} w-\left(m x_{G}-A_{35}\right) \dot{q}-\left(Z_{q}+m U\right) q$

- Linear equation of pitching motion:

$$
\begin{gathered}
M_{E}=\left(I_{y y}+A_{55}\right) \dot{q}-\left(M_{q}-m x_{G} U\right) q-\left(m x_{G}-A_{35}\right) q \\
\quad-\left(m x_{G}-A_{35}\right) \dot{w}-M_{w} w+m g\left(z_{G}-z_{B}\right) \theta
\end{gathered}
$$

In the above, $m$ refers to mass of UUV, $I_{y y}$ to mass moment of inertia about $y$-axis, $\left(x_{G}, z_{G}\right)$ to coordinates of center of gravity in $\mathrm{x}$ - and $\mathrm{z}$-direction, $z_{B}$ to $\mathrm{z}$-coordinate of center of buoyancy, $\theta$ to pitch angle, $g$ to acceleration of gravity, and $A_{i j}$ to added-mass tensor (Newman, 1978) Subscripts $i$ and $j$ in $A_{i j}$ represent six modes of motion. Terms $Z_{w}, Z_{q}, M_{q}$ and $M_{w}$ are linear hydrodynamic derivatives.

\section{Analysis Procedure}

The Fourier analysis is adopted as the method to determine derivatives. The VPMM separates the motions of a body moving through a fluid into two hydrodynamic motions, which are pure heaving and pure pitching motion. In the pure heaving case the in-phase component of force is directly related to the linear acceleration and, therefore, can be used to compute 
explicitly the associated acceleration derivatives. Similarly, in the pure pitching case the in-phase component of force is directly related to the angular velocity. Thus angular acceleration and rotary derivatives can be computed explicitly.

\section{Pure heaving motion}

Assuming the model is moving sinusoidal path, the pitch angle $\theta$, angular velocity $q$ and angular acceleration $\dot{q}$ are zero at all times since the phase angle between the struts is zero.

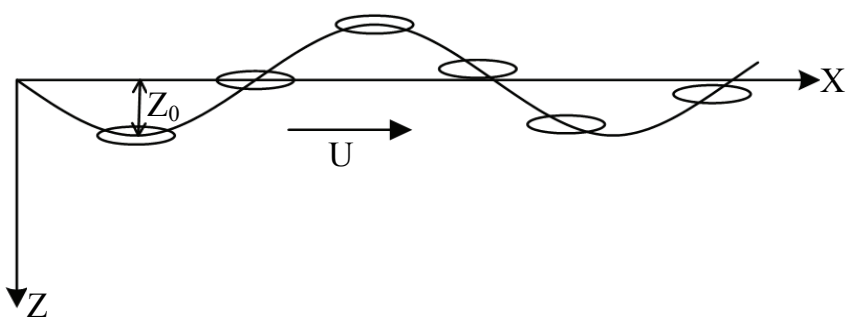

Fig. 4 Pure heaving motion.

As shown in Fig. 4, the vertical displacement $z$, linear vertical velocity $w$ and linear vertical acceleration $\dot{w}$ for pure heaving motion can be expressed respectively as

$$
\begin{aligned}
& z=z_{0} \sin \omega t \\
& w=\dot{z}=z_{0} \omega \cos \omega t \\
& \dot{w}=\ddot{z}=-z_{0} \omega^{2} \sin \omega t
\end{aligned}
$$

Where, $z_{0}$ is the amplitude of heaving motion. Using the above pure heaving conditions $(\theta=q=\dot{q}=0)$ the reduced equations for the pure heaving motion can be derived from Eq. (4) and (5).

$$
\begin{aligned}
& Z_{E}=-\left(m+A_{33}\right) \dot{w}+Z_{w} w \\
& M_{E}=-\left(m x_{G}-A_{35}\right) \dot{w}-M_{w} w
\end{aligned}
$$

Substituting Eq. (6) into the reduced equations, the equations for pure heaving motion can be written as

$$
\begin{aligned}
Z_{E}= & -\left(m+A_{33}\right)\left(-z_{0} \omega^{2} \sin \omega t\right)+Z_{w}\left(z_{0} \omega \cos \omega t\right) \\
M_{E}= & -\left(m x_{G}-A_{35}\right)\left(-z_{0} \omega^{2} \sin \omega t\right) \\
& -M_{w}\left(z_{0} \omega \cos \omega t\right)
\end{aligned}
$$

The external force and moment, $Z_{E}$ and $M_{E}$, measured during the test can be decomposed into in-phase and outphase components with the motion by Fourier analysis.

$$
\begin{aligned}
& Z_{E}=Z_{\text {in }} \sin \omega t+Z_{\text {out }} \cos \omega t \\
& M_{E}=M_{\text {in }} \sin \omega t+M_{\text {out }} \cos \omega t
\end{aligned}
$$

Therefore, hydrodynamic derivatives for pure heaving motion can be expressed as

$$
\begin{aligned}
& m+A_{33}=\frac{Z_{\text {in }}}{z_{0} \omega^{2}} \\
& Z_{w}=\frac{Z_{\text {out }}}{z_{0} \omega} \\
& m x_{G}-A_{35}=\frac{M_{\text {in }}}{z_{0} \omega^{2}} \\
& M_{w}=-\frac{M_{\text {out }}}{z_{0} \omega}
\end{aligned}
$$

\section{Pure pitching motion}

The condition that must be satisfied to obtain a pure pitching motion for a body moving through a fluid is that the pitch angle varies with time while the angle of attack $\alpha$, measured at the C.G., is maintained equal to zero at all times. The motion is one in which the body C.G. moves in sinusoidal path, with the longitudinal body axis tangent to the path, as shown in Fig. 5.

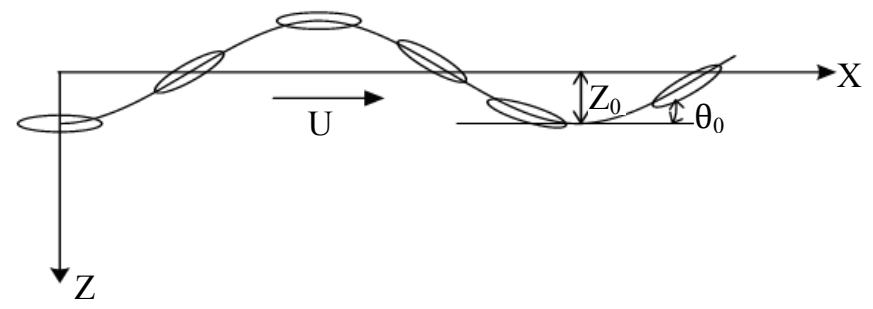

Fig. 5 Pure pitching motion.

For this condition, the resultant linear velocity $w$ and acceleration $\dot{w}$ are zero. The pitch angle $\theta$, pitching velocity $q$, and pitching acceleration $\dot{q}$ can be expressed as

$$
\begin{aligned}
\theta & =\frac{z_{2}-z_{1}}{l}=-\left(\frac{2 z_{0}}{l} \sin \frac{\phi_{s}}{2}\right)\left[\cos \left(\omega t-\frac{\phi_{s}}{2}\right)\right] \\
& =-\theta_{0} \cos \left(\omega t-\frac{\phi_{s}}{2}\right)
\end{aligned}
$$

Substituting $\omega \tau=\left(\omega t-\omega t_{1}\right)$

$\theta=\theta_{0} \sin \omega \tau$

$q=\dot{\theta}=\theta_{0} \omega \cos \omega \tau$

$\dot{q}=\ddot{\theta}=-\theta_{0} \omega^{2} \sin \omega \tau$

$\omega t_{1}=\frac{1}{2}\left(\phi_{s}+\pi\right)$

Where, $\theta_{0}$ is amplitude of pitching motion, $z_{1}$ and $z_{2}$ are amplitude of heaving motion at fore and aft, respectively, and $\phi_{s}$ is the phase difference between the fore and aft.

$z_{1}=z_{0} \sin \omega t, z_{2}=z_{0} \sin \left(\omega t-\phi_{s}\right)$

$\theta_{0}=-\frac{2 z_{0}}{l} \sin \frac{\phi_{s}}{2}$ 
Similarly, using the above pure pitching conditions ( $z=w=\dot{w}=0$ ) the reduced equations for the pure pitching motion can be derived from Eq. (4) and (5).

$Z_{E}=\left(m x_{G}-A_{35}\right) \dot{q}+\left(Z_{q}+m U\right) q$

$M_{E}=\left(I_{y y}+A_{55}\right) \dot{q}-\left(M_{q}-m x_{G} U\right) q$

Substituting Eq. (12) into the reduced equations, the equations for pure pitching motion can be written as

$$
\begin{aligned}
Z_{E} & =\left(m x_{G}-A_{35}\right)\left(-\theta_{0} \omega^{2} \sin \omega t\right) \\
& +\left(Z_{q}+m U\right)\left(\theta_{0} \omega \cos \omega t\right) \\
M_{E} & =\left(I_{y y}+A_{55}\right)\left(-\theta_{0} \omega^{2} \sin \omega t\right) \\
& -\left(M_{q}-m x_{G} U\right)\left(\theta_{0} \omega \cos \omega t\right)
\end{aligned}
$$

The external force and moment, $Z_{E}$ and $M_{E}$, is same as the pure heaving case. As the pure heaving case, therefore, hydrodynamic derivatives for pure pitching motion can be expressed as

$$
\begin{aligned}
& m x_{G}-A_{35}=-\frac{Z_{\text {in }}}{\theta_{0} \omega^{2}} \\
& Z_{q}+m U=\frac{Z_{\text {out }}}{\theta_{0} \omega} \\
& I_{y y}+A_{55}=-\frac{M_{\text {in }}}{\theta_{0} \omega^{2}} \\
& M_{q}-m x_{G} U=-\frac{M_{\text {out }}}{\theta_{0} \omega}
\end{aligned}
$$

\section{THEORETICAL CALCULATION}

Fortunately, the added mass derivatives for a spheroid can be treated with its symmetry. Consider an ellipsoid totally submerged and with the origin at the center of the ellipsoid, describe as (Thor, 1994)

$\frac{x^{2}}{a^{2}}+\frac{y^{2}}{b^{2}}+\frac{z^{2}}{c^{2}}=1$

A prolate spheroid is obtained by letting $b=c$ and $a>b$. Introduce Lamb's $k$-factors as:

$$
\begin{aligned}
& k_{1}=\frac{\alpha_{0}}{2-\alpha_{0}} \\
& k_{2}=\frac{\beta_{0}}{2-\beta_{0}} \\
& k^{\prime}=\frac{e^{4}\left(\beta_{0}-\alpha_{0}\right)}{\left(2-e^{2}\right)\left[2 e^{2}-\left(2-e^{2}\right)\left(\beta_{0}-\alpha_{0}\right)\right]}
\end{aligned}
$$

Where, $\alpha_{0}$ and $\beta_{0}$ are constants that describe the relative proportions of the spheroid.

$$
\begin{aligned}
& \alpha_{0}=\frac{2\left(1-e^{2}\right)}{e^{2}}\left(\frac{1}{2} \ln \frac{1+e}{1-e}-e\right) \\
& \beta_{0}=\frac{1}{e^{2}}-\frac{1-e^{2}}{2 e^{2}} \ln \frac{1+e}{1-e}
\end{aligned}
$$

Where, $e$ is the eccentricity of the meridian elliptical section and can be written as

$e^{2}=1-(b / a)^{2}$

Lamb and Sir Horace (1945) give representation of added mass derivatives for spheroid by defining Lamb's $k$-factors.

$$
\begin{aligned}
& X_{\dot{u}}=-k_{1} m \\
& Y_{\dot{v}}=Z_{\dot{w}}=-k_{2} m \\
& N_{\dot{r}}=M_{\dot{q}}=-k^{\prime} I_{y y}
\end{aligned}
$$

Where, the mass and the moment of inertia for a prolate spheroid are respectively.

$$
\begin{aligned}
& m=\frac{4}{3} \pi \rho a b^{2} \\
& I_{y y}=I_{z z}=\frac{4}{15} \pi \rho a b^{2}\left(a^{2}+b^{2}\right)
\end{aligned}
$$

The values of $k_{1}, k_{2}$ and $k^{\prime}$ are plotted in Fig. 6.

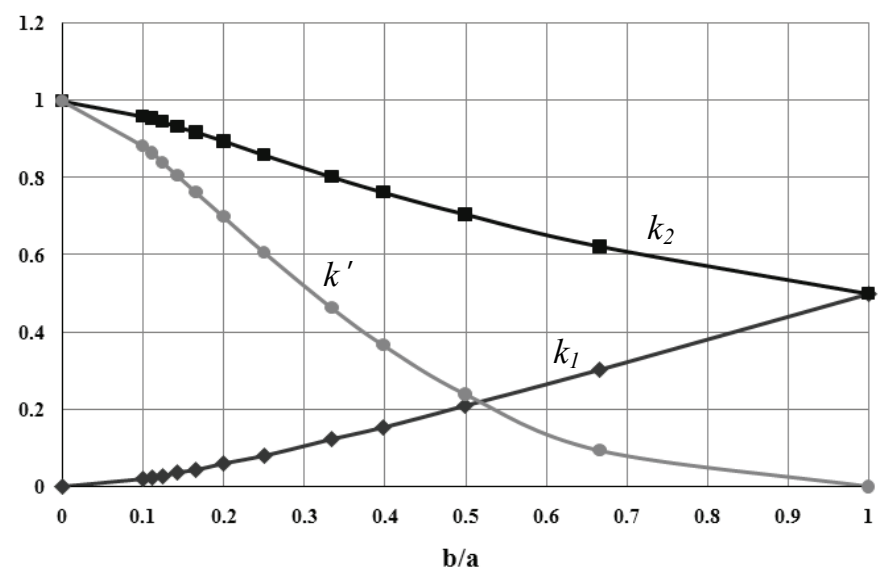

Fig. 6 Lamb's $k$-factors.

\section{CFD ANALYSIS}

To verify the results from theoretical calculation, commercial CFD code (ANSYS-CFX, 2009) is used. The elements, nodes and number of elements used for CFD analysis are shown in Table 2. 
Table 2 Principal conditions employed in the CFD analysis.

\begin{tabular}{|c|c|}
\hline Governing Equations & $\begin{array}{l}\text { Reynolds Averaged Navier- } \\
\text { Stokes (RANS) equations } \\
\text { (incompressible fluid) }\end{array}$ \\
\hline Turbulence model & $\begin{array}{l}k-\varepsilon \text { model, } \\
\text { Shear Stress Transport (SST) }\end{array}$ \\
\hline Reynolds number & Approx. 1.7563e6 \\
\hline Total no. of elements & 131,830 \\
\hline Total no. of nodes & 26,628 \\
\hline No. of Tetrahedral & 122,946 \\
\hline No. of Prisms (for B.C.) & 8,764 \\
\hline No. of Pyramids & 120 \\
\hline
\end{tabular}

\section{Mesh Generation}

The 3D model of the UUV was modeled by ANSYSDesign Modeler and exported to ANSYS-ICEM-CFD and meshed to generate the nodes and elements. 'Tetrahedral' and 'Pyramid' meshes are employed for generating nodes and elements in the fluid domain. The 'Tetrahedral' and 'Pyramid' meshes are suitable for representation of a complex geometry, but such meshes are not suitable to resolve the boundary layer adjacent to the solid body (Nishi, 2007). Therefore, 'Prism' meshes, which are the most appropriate element for a boundary layer (ANSYS-CFX, 2007), are used for generating elements in the boundary layer around the body.

Fig. 7 shows the various meshed sections (hybrid mesh) which are merged, and embodied for the CFD analysis by the "ANSYS-CFX-MESH" mesh generator.

\section{Turbulence Model}

Various turbulence models, such as $k-\varepsilon$, Shear Stress Transport, BSL Reynolds Stress, SSG Reynolds Stress model, were proposed to provide solutions to the Reynolds stresses in terms of known quantities to allow closure of the RANS by ANSYS CFX. From the various turbulence models, $k-\varepsilon$ model and Shear Stress Transport (SST) were considered in this study. The reason for this selection is that the $k-\varepsilon$ model is a commonly used turbulence model for engineering simulations due to its robustness and application to a wide range of flows, while the Shear Stress Transport model is better at predicting separation (ANSYS-CFX, 2007) likely to be found at the aft of the UUV.

\section{Description of UUV's Motion}

For the VPMM simulation, the motion of the UUV moving sine wave should be simulated. The "MESHDEFORMAION" was adopted for the motion simulation that the UUV moves the calculated position by the equation according to the time. The concerned domain moves with considering the UUV motion itself by the "MESHDEFORMAION" in the simulation. Because of the reason that the concerned domain moves, the meshes in the domain should not affect to the CFD results due to the change of the size or shape of the meshes. In this study, the position of the UUV was specified for the similar motion with the experimental test movement by using ANSYS-CFX Command Language (CCL).
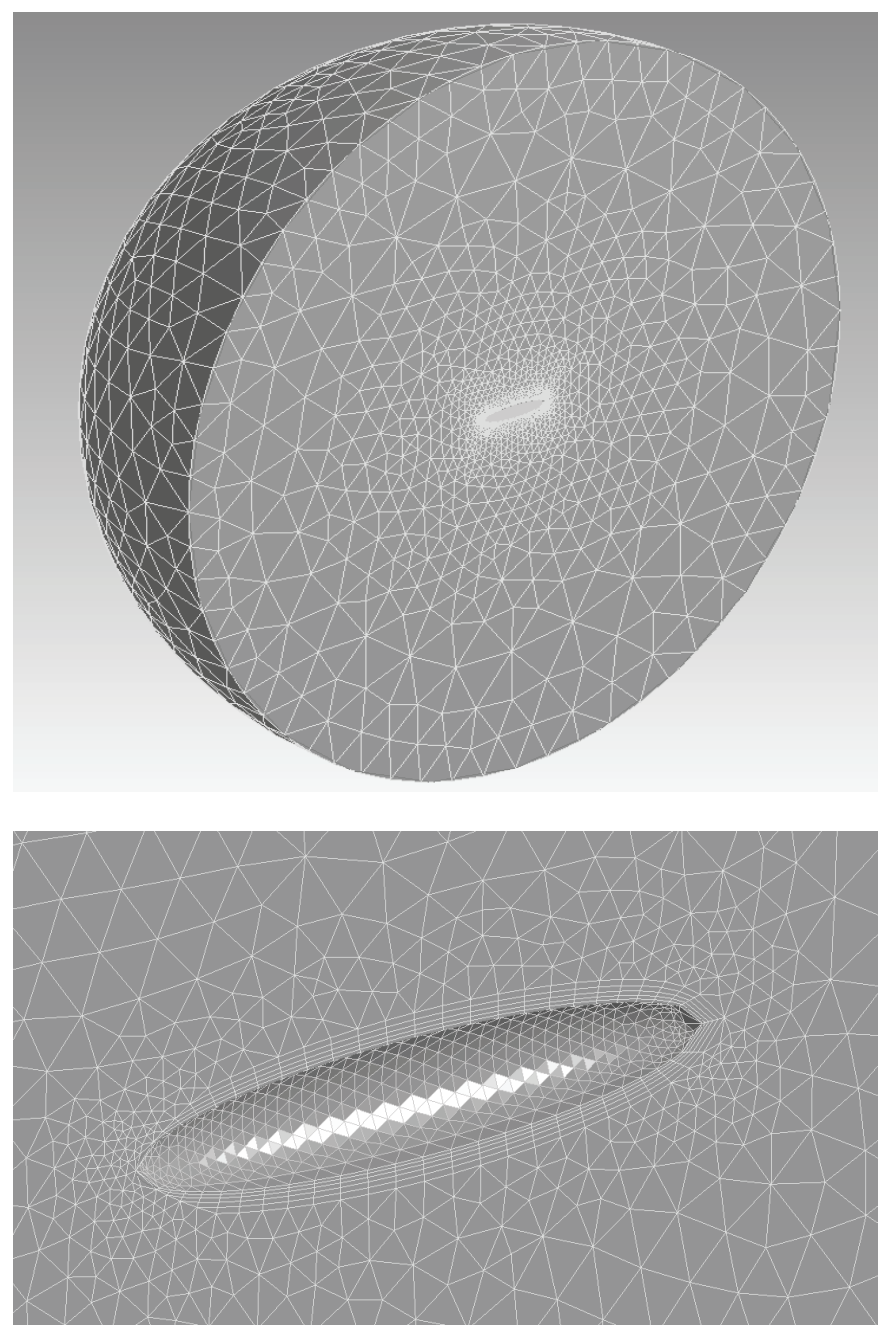

Fig. 7 Hybrid mesh (Structured and unstructured mesh) generated adjacent to the main body of the UUV.

Fig. 8 and 9 show the pressure and velocity distribution around the UUV when it moves to the traces of the Pure Heave and Pitch motions respectively. The pressure distribution around the AUV (seen in Fig. 8) shows the maximum pressure approx. $436 \sim 441 \mathrm{~Pa}$ ) occurs in the bows, but an even distribution fore and aft of the hull. Along the mid-body, the boundary layer grows and the flow is accelerated as it reaches the stern. Any vertical structure has not been form behind the stern as shown in Fig. 9 due to its simple shape and low velocity (or low Reynolds no.).

The $\mathrm{z}$-axis direction forces for the Pure Heave Motion and Pure Pitch Motion from VPMM test and CFD analysis are compared in Fig. 10 and 11. The z-axis direction force predictions from the experimental test and CFD results have a high degree of correspondence as shown in Fig. 10 and Fig. 11. Most of the cases, the CFD values are the mean values of the experimental test results from the towing tank. 


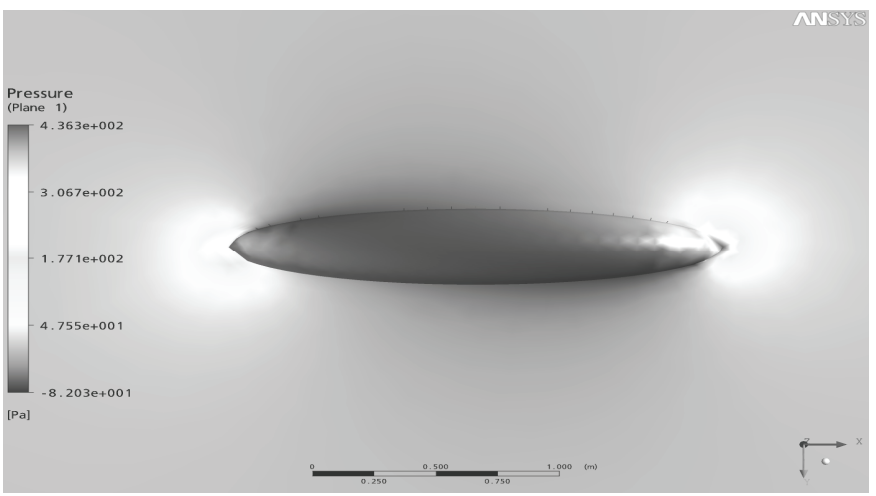

(a) Pure Heave Motion (@T=10)

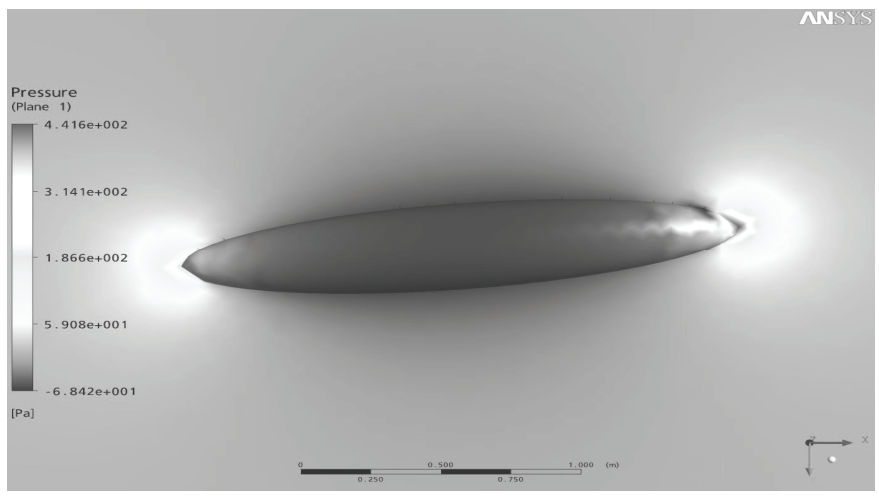

(b) Pure Pitch Motion (@T=10)

Fig. 8 Pressure distribution around the UUV.

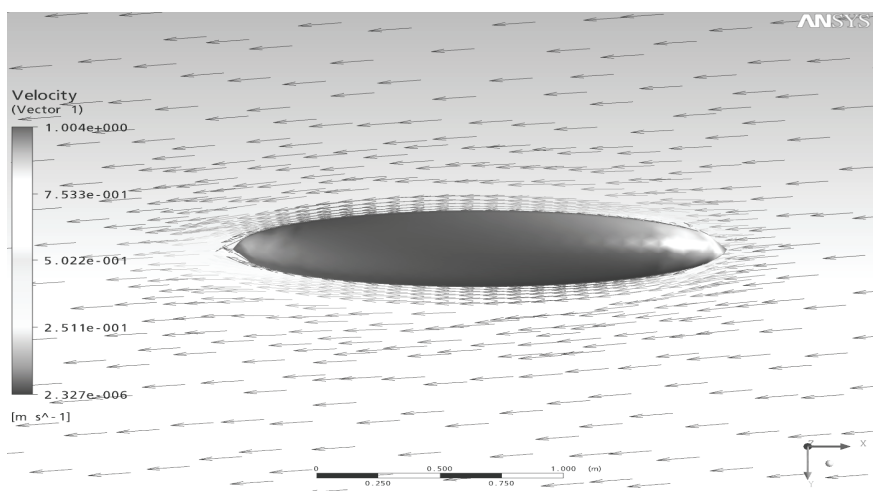

(a) Pure Heave Motion (@T=10)

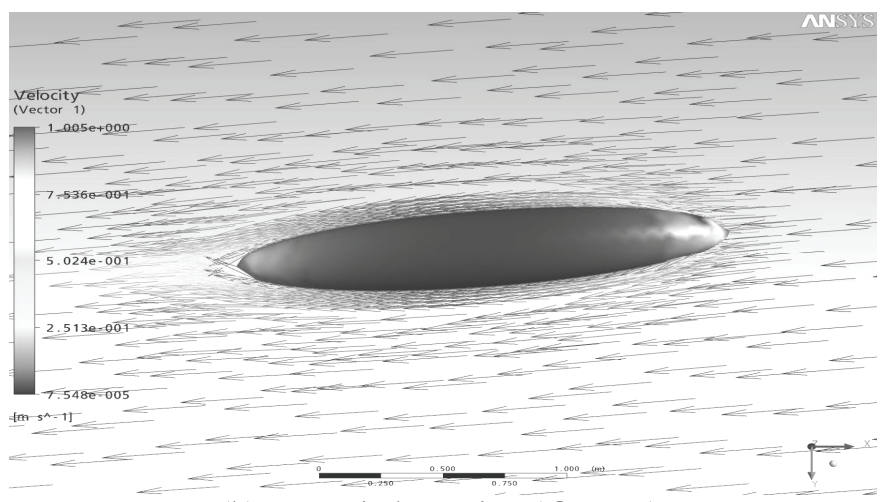

(b) Pure Pitch Motion (@T=10)

Fig. 9 Velocity distribution around the UUV.
Table 3 Comparison of results.

\begin{tabular}{|c|c|c|c|}
\hline \multirow{2}{*}{$\begin{array}{c}\text { Pure heaving } \\
\text { motion }\end{array}$} & \multicolumn{3}{|c|}{ Added mass } \\
\cline { 2 - 4 } & VPMM test & $\begin{array}{c}\text { CFD } \\
\text { analysis }\end{array}$ & $\begin{array}{c}\text { Theoretical } \\
\text { Cal. }\end{array}$ \\
\hline $\mathrm{T}=5 s$ & $1.0667 m$ & $0.9907 m$ & $0.9066 m$ \\
\hline $\mathrm{T}=8 s$ & $1.1112 m$ & $1.0780 m$ & $0.9066 m$ \\
\hline $\mathrm{T}=10 s$ & $1.2434 m$ & $1.1240 m$ & $0.9066 m$ \\
\hline \multirow{2}{*}{$\begin{array}{c}\text { Pure pitching } \\
\text { motion }\end{array}$} & \multicolumn{3}{|c|}{ Added inertia } \\
\cline { 2 - 4 } & VPMM test & $\begin{array}{c}\text { CFD } \\
\text { analysis }\end{array}$ & $\begin{array}{c}\text { Theoretical } \\
\text { Cal. }\end{array}$ \\
\hline $\mathrm{T}=10 s$ & $0.7769 I_{y y}$ & $\cdot$ & $0.7335 I_{y y}$ \\
\hline $\mathrm{T}=12 s$ & $1.5882 I_{y y}$ & $\cdot$ & $0.7335 I_{y y}$ \\
\hline
\end{tabular}

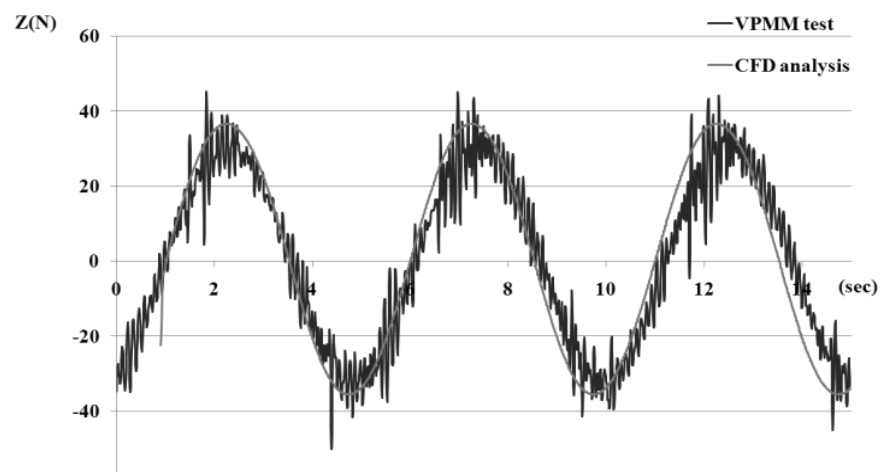

(a) $\mathrm{T}=5 \mathrm{sec}$

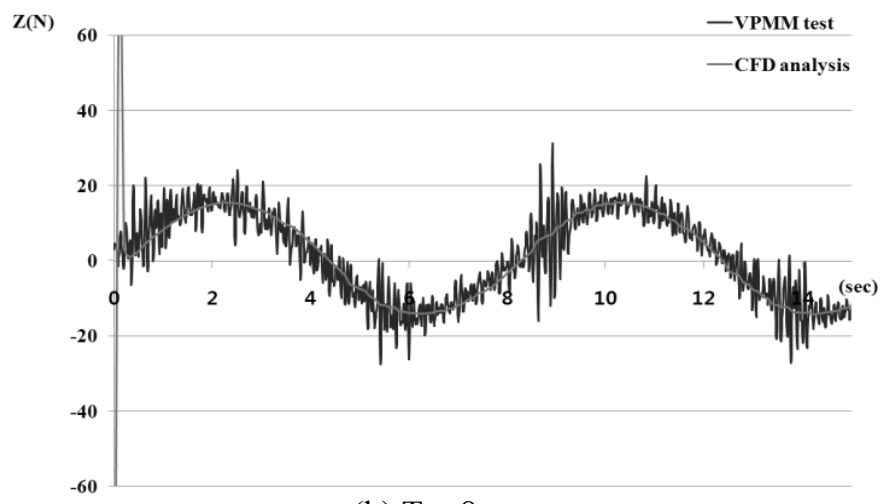

(b) $\mathrm{T}=8 \mathrm{sec}$

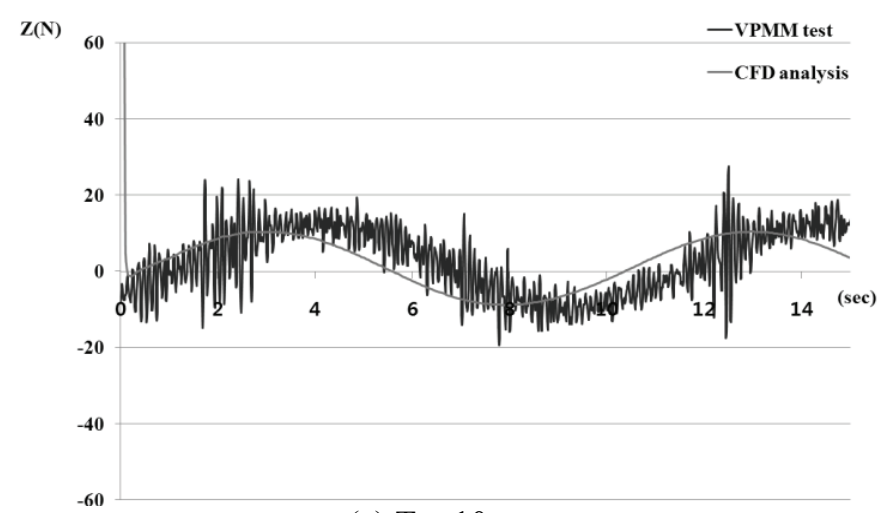

(c) $\mathrm{T}=10 \mathrm{sec}$

Fig. 10 Comparison of the experimental and CFD results for the $\mathrm{Z}$-force of the Pure Heaving Motion. 


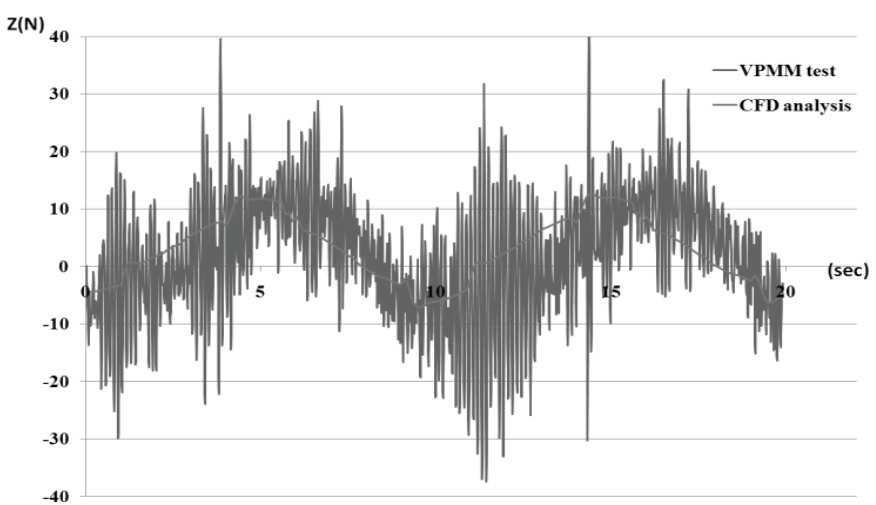

(a) $\mathrm{T}=10 \mathrm{sec}$

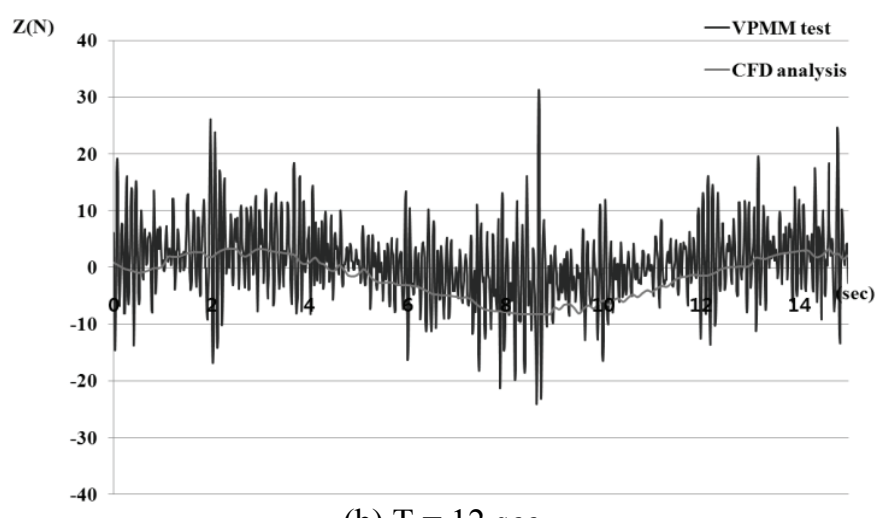

(b) $\mathrm{T}=12 \mathrm{sec}$

Fig. 11 Z-force of the Pure Pitch Motion.

Conclusively, the added mass values obtained from the pure heave motion CFD analysis are 0.9907m (@ T=5s), 1.0780m (@T=8s),1.1240m (@ T=10s), and that value has a high degree of agreement with the experimental test (VPMM test) result in the towing tank as shown in Table 3.

\section{RESULTS}

As a result of theoretical calculation, the added mass and added inertia were $0.9066 \mathrm{~m}$ and $0.7335 I_{y y}$, respectively. In VPMM test, they were $1.1206 m$ and $0.1527 I_{y y}$ on the average. Excepting for lower frequencies ( $\mathrm{T}=8,10 \mathrm{~s}$ in pure heaving case and $\mathrm{T}=10,12 \mathrm{~s}$ in pure pitching case), the added mass and the added inertia showed no significant difference between the experimental results and theoretical calculations. That is, when the forced oscillating period are $5 s$ (in pure heaving motion) and $10 s$ (in pure pitching motion), the added mass and the added inertia were $1.0667 \mathrm{~m}$ and $0.7769 I_{y y}$.

\section{CONCLUSIONS}

In this paper, the added mass and inertia derivatives obtained by VPMM test, theoretical calculation and CFD analysis are described for a spheroid-type UUV. As results, the validity of the equipment for VPMM test can be verified with a sufficient accuracy. Results with longer period have some errors and, therefore if possible, tests at the shortest period of the forced oscillation are desirable.

\section{ACKNOWLEDGEMENTS}

This research was supported by UVRC (Underwater Vehicle Research Center) and UTRC (Unmanned Technology Research Center) of ADD (Agency for Defense Development), and this work was supported by the National Research Foundation of Korea Grant funded by the Korean Government (Ministry of Education, Science and Technology) [NRF-2010-355-D00082]. Their financial support is gratefully acknowledged.

\section{REFERENCES}

Abkowitz, M.A., 1969. Stability and Motion Control of Ocean Vehicles, The MIT Press, Cambridge. pp.346.

Lamb, S.H., 1945. Hydrodynamics, Sixth Edition, Dover Publications, pp.152-155.

Newman, J.N., 1978. Marine Hydrodynamics, The MIT Press, Cambridge, pp.144-148.

Nishi, Y. Kashiwagi, M. Koterayama, W. Nakamura, M. Samuel, S.Z.H. Yamamoto, I. and Hyakudome, T., 2007. Resistance and Propulsion Performance of an Underwater Vehicle Estimated by a CFD Method and Experiment, ISOPE '07, Lisbon, Spain.

Shon, K.H. Lee, S.K. and Ha, S.P., 2006. Mathematical Model for Dynamics of Manta-type Unmanned Undersea Vehicle with Six Degrees of Freedom and Characteristics of Manoeuvrability Response, Journal of the Society of Naval Architects of Korea, 43(4), pp.399-413.

Thor, I.F., 1994. Guidance and Control of Ocean Vehicles, John Wiley and Sons, pp.37-42. 